\title{
Anti-HER2/Anti-HER3 Bispecific Monoclonal Antibody MCLA-128
}

National Cancer Institute

\section{Source}

National Cancer Institute. Anti-HER2/Anti-HER3 Bispecific Monoclonal Antibody MCLA128. NCI Thesaurus. Code C153211.

A full-length IgG1 bispecific antibody with enhanced antibody-dependent cellular cytotoxicity (ADCC) directed against human epidermal growth factor receptor 2 (HER2; EGFR2, ERBB2) and human epidermal growth factor receptor 3 (HER3; ErbB3), with potential antineoplastic activity. Upon intravenous administration, the bispecific antibody docks on HER2, and subsequently blocks heregulin-stimulated proliferation of tumor cells by binding HER3. In addition to inhibiting HER3-dependent signaling, simultaneous targeting of HER2 and HER3 by MCLA-128 may overcome a common resistance mechanism driven by heregulin-mediated dimerization of HER2 and HER3. MCLA-128 is expected to eliminate tumor cells by recruiting natural killer (NK) cells to tumor cells coated with the bispecific antibody. 